\title{
The Effect of Crystalloid Co-Loading on Circulatory Stability During General Anaesthesia Induction in ASA II/III Patients for Major Abdominal Surgery: A Randomized Controlled Trial
}

\section{Darjan Kos ( $\nabla$ k.darjan@gmail.com )}

UKC Maribor: Univerzitetni Klinicni Center Maribor https://orcid.org/0000-0003-3232-9356

Mirt Kamenik

UKC Maribor: Univerzitetni Klinicni Center Maribor

Dejan Dinevski

Univerza v Mariboru Medicinska fakulteta

\section{Research}

Keywords: Circulatory stability, co-loading, anaesthesia induction

Posted Date: February 18th, 2022

DOI: https://doi.org/10.21203/rs.3.rs-1272136/v1

License: (c) (i) This work is licensed under a Creative Commons Attribution 4.0 International License.

Read Full License 


\section{Abstract}

\section{Background}

We studied the effect of crystalloid co-loading with simultaneous infusion of vasopressor phenylephrine on circulatory stability during general anaesthesia induction with propofol in ASA II/III patients. We analysed the postinduction phase because circulatory instability is most prevalent in this period and more prominent in multimorbid patients. Prevention rather than treatment might be a better strategy to avoid complications associated with even short periods of instability.

\section{Methods}

46 ASA II/III patients were randomly assigned to two treatment groups. During the induction, the control group received an infusion of phenylephrine only and the test group received the same phenylephrine regimen and also a crystalloid co-load. Cardiac index (Cl), mean arterial pressure (MAP), stroke volume index (SVI), systemic vascular resistance index (SVRI), stroke volume variation (SVV) and heart rate (HR) were recorded for 18 minutes and the dynamics of change between groups analysed.

\section{Results}

$\mathrm{Cl}$ decreased in both groups after the induction but less in the test group, almost reaching statistical significance $(p=0,052)$. MAP also decreased more in the control group; however, not reaching statistical significance. HR decreased in both groups, significantly more in the test group $(p=0,041)$. SVRI almost identically increased in both groups. SVI decreased in the control group, whereas it significantly increased after initial decrease in the test group $(p=0,006)$. SVV slowly decreased under $10 \%$ in the test group and increased over $10 \%$ in the control group.

\section{Conclusions}

Our results show that phenylephrine combined with crystalloid co-loading enables better circulatory stability than phenylephrine alone.

\section{Trial registration}

The study was registered on $15^{\text {th }}$ of March 2017 at ISRCTN registry: http://www.isrctn.com/ISRCTN46293288 and was retrospectively registered.

Clinical trial number is ISRCTN46293288.

\section{Background}

Circulatory stability during anaesthesia is one of the most important tasks for the anaesthesiologist. Propofol as the most used induction agent enables smooth anaesthesia induction and provides the most satisfying intubating conditions. However, induction with propofol is often associated with hypotension 
and a decrease in cardiac index $(\mathrm{Cl})$. These changes usually do not have a significant impact on adverse outcomes in otherwise healthy patients. But elderly patients with comorbidities are more prone to circulatory instability after the induction $(1,2)$. The incidence of hypotension and decrease in cardiac index $(\mathrm{Cl})$ is significantly higher in this group of patients (3). Perioperative hypotension is associated with major postoperative complications, including central nervous ischemia and stroke (4), myocardial injury, acute kidney injury $(5,6)$ and death $(7)$. Even short durations of intraoperative mean arterial pressure (MAP) values less than $65 \mathrm{mmHg}$ or values below $20 \%$ the preoperative baseline was independently associated with organ damage $(6,7,8)$.

Maintaining blood pressure during the induction does not guarantee a normal flow. $\mathrm{Cl}$ and blood pressure are not interchangeable physiological variables. Normal or even high pressure does not necessarily mean a normal flow and hypotension does not necessarily mean a low flow. Intraoperative stroke volume index $(\mathrm{SVI})$ and $\mathrm{Cl}$ optimisation is associated with a better outcome $(9,10,11)$. For these reasons, our goal during the perioperative period is to maintain blood pressure and $\mathrm{Cl}$. However, cardiac output is rarely monitored during surgery.

Preventing circulatory instability during the induction of general anaesthesia in elderly multimorbid patients should be a preferred approach to simply treating it. Vasopressors and fluid therapy are therapeutic interventions of choice. In clinical practice we usually use phenylephrine, a selective a1 agonist. Our previous study (12) has shown that a phenylephrine infusion during anaesthesia induction with propofol results in a more stable MAP compared to the control group. But the phenylephrine infusion did not prevent the decrease in $\mathrm{Cl}$ after induction, although it did show a slightly improved preload.

Fluid optimisation and normovolemia are used to enable adequate preload and consequently SVI. There is no universal definition of normovolemia and no universal protocol of intravenous fluid regimen exists. Fluid therapy is aimed at correcting relative hypovolemia due to the vasodilatory effects of anaesthetics. A bolus of crystalloids has a short volume effect. Continuous infusion enables more gradual distribution and better volume effect compared to bolus (13). Distribution is also more gradual during the induction because of vasodilation and consequently lower filtration pressure (14). By using an optimal co-loading during the induction in addition to vasopressors, we should be able to maintain pressure and flow.

Literature review has not shown any study evaluating the haemodynamic effects of a continuous infusion of phenylephrine combined with co-loading with crystalloids during the induction of general anaesthesia with propofol in ASA II/III patients undergoing major abdominal surgery. We hypothesized that combined therapy would improve haemodynamics in high-risk patients, by maintaining MAP and $\mathrm{Cl}$ during the induction. We also compared stroke volume variation (SVV) between groups to determine how co-loading simultaneously with vasopressor infusion would impact fluid responsiveness. Additionally, we aimed to evaluate the correlation between fluid responsiveness, $\mathrm{Cl}$ and MAP. $\mathrm{Cl}$ was taken as the primary outcome variable and MAP, heart rate (HR), SVV, systemic vascular resistance index (SVRI) and SVI were taken as secondary outcome variables. 


\section{Methods}

ASA II/III patients scheduled for major abdominal surgery were included. A signed informed consent was obtained from each patient. The exclusion criteria were ejection fraction of less than $30 \%$, moderate or severe valvular heart disease, acute or chronic renal failure with serum creatinine higher than $120 \mathrm{mmol} / \mathrm{l}$, body mass index more than 30 , and anticipated difficult intubation.

Patients were fasting overnight. On the morning of surgery, they took their regular medication, except ACE inhibitors or angiotensin receptor blockers (ARBs) and were premedicated with midazolam $0,1 \mathrm{mg} / \mathrm{kg}$ orally. Upon arrival in the operating room an intravenous and an arterial line were placed and the BIS electrodes were attached to monitor the depth of anaesthesia. The arterial line was connected to the LiDCOrapid monitor (LiDCO Cardiac Sensor Systems, Cambridge, UK) to enable SVI, CI, SVV and SVRI measurement in addition to invasive MAP monitoring. Patients were randomly assigned to the treatment and control group according to the crystalloid infusion protocol. The control group received a maintenance infusion of $4 \mathrm{ml} / \mathrm{kg} / \mathrm{h}$ and the treatment group received $10 \mathrm{ml} / \mathrm{kg}$ infused during the time the measurements were recorded.

Baseline values were taken the first minute of measurements. Then fentanyl bolus $3 \mathrm{mcg} / \mathrm{kg}$ was administered and after two minutes the propofol infusion $0,5 \mathrm{mg} / \mathrm{kg} / \mathrm{min}$ was started. Simultaneously, we initiated the phenylephrine infusion $0,5 \mathrm{mcg} / \mathrm{kg} / \mathrm{min}$ in both groups and a crystalloid infusion according to the patient group protocol. When the bispectral index (BIS) value reached 60, propofol infusion was stopped and rocuronium $1 \mathrm{mg} / \mathrm{kg}$ bolus was administered. One minute after rocuronium administration, the patients were intubated and mechanically ventilated with a tidal volume of $8 \mathrm{ml} / \mathrm{kg}$. They received air/oxygen mixture with sevoflurane to maintain BIS values between 40 and 60 .

$\mathrm{Cl}, \mathrm{MAP}, \mathrm{HR}, \mathrm{SVI}, \mathrm{SVRI}$ and SVV were recorded continuously for 18 minutes before and during the induction. Measurements recorded were averaged for each minute, the first minute representing preinduction or baseline values. We also recorded the time interval between the beginning of induction until intubation.

We aimed to maintain MAP between 65 and baseline $\mathrm{mmHg}$ values and heart rate between 40 and 90 beats per minute. If MAP increased above baseline values, we stopped the infusion of phenylephrine. Persisting hypertension was treated with three additional boluses of fentanyl ( $3 \mathrm{mcg} / \mathrm{kg}$ ) and if not efficient, a nitro-glycerine infusion was applied. Hypotension lasting more than one minute was treated with additional boluses of phenylephrine $50 \mathrm{mcg}$. Tachycardia was managed with a maximum of three additional boluses of fentanyl $(3 \mathrm{mcg} / \mathrm{kg}$ ). Bradycardia was treated with atropine $0,3 \mathrm{mg}$ three times and, if combined with hypotension, additionally with ephedrine $5 \mathrm{mg}$ until improvement.

Data were analysed with the IBM SPSS Statistics 27 statistical software. Data were tested for normality using the Kolmogorov-Smirnov test. The patients' characteristics and baseline values were compared using a t-test for independent samples or the Mann-Whitney test, and $\chi^{2}$ or Fisher exact test where appropriate. The analysis of variance combined with linear regression or analysis of repeated measures 
covariance (ANCOVA), respectively, was performed to compare each haemodynamic parameter. P-value < 0.05 was considered as statistically significant.

Sample size calculation to detect a difference in $\mathrm{Cl}$ of $0.2 \mathrm{l} / \mathrm{min} / \mathrm{m}^{2}\left(\mathrm{SD} 0.15 \mathrm{l} / \mathrm{min} / \mathrm{m}^{2}\right)$ among treatment groups with a probability level of 0.05 and power of 0.90 yielded a sample size of 12 patients for each treatment group.

\section{Results}

We randomized 46 patients. 7 were excluded from the analysis due to technical issues with monitors or arterial catheter and difficult intubation. 39 patients were included in the analysis. A flowchart of the study conduct is included (Figure 1 - Flowchart of the conduct of the study). There were no significant differences between groups regarding patient demographics, comorbidities, baseline haemodynamic measurements and mean dose of propofol (Table 1). Haemodynamic data for both study groups are shown in Table 2 and graphically in Figure 2 - Haemodynamic data development for both study groups. Blue - Control group, Red - Test group.

During the induction of anaesthesia and before intubation (time interval 1-6 minutes) MAP, Cl, HR SVI decreased compared to baseline values. SVRI increased slightly, while SVV fluctuated insignificantly around the baseline values.

There were no significant differences between the groups regarding MAP, Cl, HR, SVRI, SVI and SVV dynamics in the time interval 1-6 minutes. (Table 2, Figure 2).

After intubation (time interval 6-9 minutes), MAP increased in both groups of patients, significantly more in the test group. MAP slowly decreased afterwards in both groups in the time interval 9-18 minutes, less in the test group. However, the difference in MAP decrease was not significant between groups.

HR increased after intubation in both groups and then slowly decreased. The change was not significant between groups in time intervals 6-9 and 9-18 minutes. However, a significant change between groups was determined in the time interval 1-18 minutes, because the baseline value was slightly higher in the test group.

SVI dynamics were significant in time intervals 1-9, 6-9 and 1-18 minutes. SVI decreased in both groups until intubation. After intubation, it continued to slowly decrease in the control group, contrary to the test group where it increased.

$\mathrm{Cl}$ decreased slowly in both groups of patients, more in the control group. Dynamics almost reached significance in the time interval 1-18 minutes ( $p$-value $=0.052$ ).

SVV change was the most significant in time intervals 1-9 and 6-9 minutes, when the difference in SVV value between groups became the most prominent. SVV decreased in the bolus group and increased in 
the control group compared to baseline. The dynamics were not significant between groups afterwards in the time interval 9-18 minutes. Overall, the change was significant in the time interval 1-18 minutes.

SVRI increased in both groups. However, the dynamics were not significant in any time interval between groups.

The phenylephrine infusion was stopped in 5 patients in the test group due to a sustained increase in MAP. No additional interventions to stabilize haemodynamic were necessary in this group. In the control group, three patients needed additional phenylephrine boluses due to hypotension. Phenylephrine infusion was stopped in two patients for a sustained increase in MAP and two patients in the control group received one bolus of atropine because of bradycardia.

No further haemodynamic complications were observed.

\section{Discussion}

We studied the impact on haemodynamic of co-loading with the Ringer's solution simultaneous with phenylephrine infusion during BIS-guided general anaesthesia induction with propofol in ASA II/III patients scheduled for a major abdominal surgery.

We used the uncalibrated arterial waveform analysis. Although being somewhat inaccurate regarding the absolute values of haemodynamic variables, this fact was acceptable as we were interested in the dynamics of these variables compared to baseline values.

Intraoperative hypotension ( $\mathrm{IOH})$ is probably not a uniform entity as the study suggests (15). Different pathophysiological mechanisms (phases of anaesthesia and/or surgery) underlie intraoperative haemodynamic instability, therefore $\mathrm{IOH}$ might be divided into different phases. The postinduction phase was defined as a period from the induction until 20 minutes after the induction but before the beginning of surgery. We focused on the postinduction phase, because haemodynamic instability is most prevalent in this phase and it is affected by anaesthesia and patient characteristics only.

BIS-guided protocol was used to titrate propofol to reach the desired depth of anaesthesia. Due to the of pharmacokinetic properties of propofol, BIS further decreases after stopping the infusion $(16,17)$. There is also a 10-15 seconds delay in the BIS value (18). Therefore, we stopped the propofol infusion at BIS level 60. This approach reduces the cumulative dose of propofol required for appropriate anaesthesia depth $(19,20)$.

Maintaining circulatory stability during anaesthesia is achieved by maintaining optimal MAP and $\mathrm{Cl}$. But the definition of optimal MAP and $\mathrm{Cl}$ is much less clear. According to the consensus, absolute MAP values higher than $65 \mathrm{mmHg}$ are recommended (21). However, these populational harm thresholds do not necessarily reflect an individual threshold as there is considerable variation in baseline pressure among patients. Some authors suggest that MAP should be maintained within $20 \%$ of baseline values. However, prevention of hypotension rather than treatment is probably a more reasonable approach. There are 
many studies in the literature evaluating the preventive measures to maintain MAP and $\mathrm{Cl}$ during the induction of spinal anaesthesia. Although the induction of general anaesthesia is usually accompanied with large variations in blood pressure and flow, studies evaluating the preventive measures to maintain MAP and $\mathrm{Cl}$ during the induction of general anaesthesia are rare. Our previous study (12) evaluated the impact of phenylephrine infusion during the BIS-guided general anaesthesia induction with propofol in ASA III patients scheduled for a major abdominal surgery. The study showed a significantly more stable MAP in the phenylephrine group compared to the control group. $\mathrm{Cl}$ was also higher in the phenylephrine group but the difference became significant only at the end of measurements. MAP was therefore higher predominantly because of the arterioconstriction. A minor contribution to MAP was also insignificantly higher $\mathrm{Cl}$ due to the increase in SVI. Higher SVI was explained by venoconstriction caused by phenylephrine increasing preload through changing unstressed volume to stressed volume. This explanation was probably oversimplified as a agonists have complex effects depending on many factors $(22,23,24,25)$. The study of Rebet and co-workers (26) evaluated the effects of phenylephrine even further. They have shown that the effects of phenylephrine are influenced by the preload dependency state. In the preload dependent patients, phenylephrine increased SV and CO through decreased venous capacitance and increased preload. Contrary, in preload independent patients it decreased SV and CO because its effects on the increased afterload predominated.

The speed of phenylephrine infusion in our study was within the recommended dose interval (27). We have chosen a fixed rate of infusion as there are no clear recommendations or studies concluding whether to use a fixed or variable infusion rate. There were some studies determining the infusion rate in spinal anaesthesia for caesarean section but without clear evidence supporting any (28).

In addition to maintaining MAP, we also aim to maintain the flow. According to literature evaluating the induction of spinal anaesthesia, co-loading in the form of infusion has a better volume effect than preloading or single bolus co-loading $(29,30)$. We hypothesized that crystalloid co-loading during the induction of general anaesthesia might contribute to a more preserved preload and consequently more stable $\mathrm{Cl}$ than phenylephrine alone. In our study the decrease in MAP, $\mathrm{Cl}, \mathrm{SVI}$ and $\mathrm{HR}$ after the induction and before intubation was similar in both groups of patients. The decrease in MAP, SVI and Cl was caused by the venodilator properties of propofol and consequently blood pooling with a decrease in preload, while the decrease in HR was caused by the opioids (31). Arteriodilation is probably less important. Intubation, being a painful stimulus, caused only a brief increase in MAP, Cl and HR. SVI in this period increased only in the test group. After intubation, MAP and $\mathrm{Cl}$ decreased less in the test group. Significantly higher SVI contributed to higher $\mathrm{Cl}$ in the test group. However, this effect was reduced because of the decreased HR due to activation of the baroreceptor reflex by higher MAP. SVRI dynamics were similar in both groups reflecting equal effects of phenylephrine on arterial vasoconstriction. SVV fluctuated around $10 \%$ before the induction in both groups, meaning they were not fluid responsive and consequently did not need additional fluid therapy. SVV increased well above $10 \%$ after intubation in the control group. According to recommendations that would mean that patients were fluid responsive and needed fluid therapy, which corelated well with SVI decrease. In the test group, SVV decreased well below $10 \%$, signifying sufficient fluid therapy. It started to slowly increase after 14 minutes of measurements, 
reflecting gradual fluid redistribution, but never increased above $10 \%$. There is some contradictory evidence about the vasopressor effect on SVV. Regarding the venous system, the phenylephrine effect opposes that of propofol, with venoconstriction changing unstressed volume to stressed volume and enhancing venous return, but this effect was equal in both groups of patients in our study.

Combined, crystalloid co-loading and phenylephrine infusion enabled more stable $\mathrm{Cl}$ and MAP than phenylephrine alone. However, both parameters decreased substantially from the baseline. Higher doses of phenylephrine would unlikely be of benefit. In both groups there were patients where MAP increased to the extent that we had to stop phenylephrine, suggesting even lower doses might be enough in some patients. On average, a higher dose of phenylephrine would increase MAP even more but simultaneously decrease $\mathrm{Cl}$ as higher SVRI increasing the afterload would probably lead to reduced $\mathrm{Cl}$ and would produce even more pronounced reflex bradycardia. Also, a favourable effect of venoconstriction, that is pooling blood to the heart, would be outweighed by higher venous resistance to flow and preload would eventually decrease (32).

It is in our interest to avoid excessive fluid administration and according to SVV, fluid therapy was sufficient in the test group and insufficient in the control group. However, SVI did not maintain baseline values in the test group. Would additional fluid boluses, regardless of SVV being less than $10 \%$, maintain SVI even better? From another perspective, considering SVV, our test group should be preload independent, meaning that phenylephrine as to the study (26) should decrease CO compared to the control group which was preload dependent. However, that was not the case, because $C O$ was eventually higher in the test group. Even though SVV was less than $10 \%$, it is possible this group of patients was still not preload independent and there might be some extra space for additional fluid regardless of $S V V<$ $10 \%$.

In our opinion relying only on SVV during the induction of anaesthesia might be insufficient to maintain haemodynamics.

There is no universal approach and therapy should be tailored to each patient individually. Combined therapy, phenylephrine and crystalloid co-loading in our study enabled more stable $\mathrm{Cl}$ and MAP during anaesthesia induction with propofol. However, it might be reasonable to think about using a vasopressor that does not induce bradycardia in the same extent as phenylephrine. Noradrenaline probably does not induce such bradycardia as phenylephrine and through $\beta 2$ agonism causes enhanced hepatic blood flow and consequently preload. But it causes more frequent rhythm disturbances and favourable $\beta$ agonism takes place at higher doses. It would be interesting to define and compare different phenylephrine dosing regimens, sole co-loading regimen and compare phenylephrine and noradrenaline during the induction to achieve optimal circulatory stability. Another possible approach to study would be using inotrope to oppose possible propofol induced cardiac depression.

A limitation of this study is a relatively small number of patients. 


\section{Conclusions}

The results of our study demonstrate that combined, continued fluid and vasopressor phenylephrine therapy enables better haemodynamic stability than phenylephrine alone. Further understanding of events necessitates additional studies because physiologic interplay between the effects of general anaesthesia and interventions like fluid and vasopressor therapy is complex.

\section{List Of Abbreviations}

$\mathrm{Cl}$

Cardiac index

MAP

Mean arterial pressure

SVV

Stroke volume variation

SVRI

Systemic vascular resistance index

$\mathrm{HR}$

Heart rate

SVI

Stroke volume index

BIS

Bispectral index

$\mathrm{IOH}$

Intraoperative hypotension

\section{Declarations}

\section{Ethics approval and consent to participate}

The study was approved by the National Medical Ethics Committee (Republic of Slovenia Medical Ethics Committee) (Ref.: 0120-241/2016-2).

\section{Consent for publication}

Not applicable

\section{Availability of data and materials}

The datasets used and/or analysed during the current study are available from the corresponding author on reasonable request.

Competing interests 
The authors declare that they have no competing interests.

\section{Funding}

The work was funded by the institution: University Medical Centre Maribor, Ljubljanska 5, 2000 Maribor, Slovenia. There were no other sources of funding.

\section{Authors' contributions}

DK was a major contributor in the design of the work, acquisition of data and in writing the manuscript. DD analyzed and interpreted the patient haemodynamic measurement data. MK substantively revised the work. All authors read and approved the final manuscript.

\section{Acknowledgements}

Not applicable.

\section{References}

1. Reich DL. Hossain S, Krol M, et al. Predictors of hypotension after induction of general anesthesia Anesth Analg 2005;101:622-8.

2. Kheterpal S. O'Reilly M, Englesbe MJ, et al. Preoperative and intraoperative predictors of cardiac adverse events after general, vascular, and urological surgery. Anesthesiology. 2009;110(1):58-66..

3. Survey of anesthesia-related mortality in France. Anesthesiology. 2006;105(6):1087-1097..

4. Bijker JB. Gelb AW. Review article: the role of hypotension in perioperative stroke. Can J Anaesth 2013;60(2):159-67.

5. Walsh M. Devereaux PJ, Garg AX, et al. Relationship between intraoperative mean arterial pressure and clinical outcomes after noncardiac surgery: toward an empirical definition of hypotension. Anesthesiology. 2013;119(3):507-515..

6. Salmasi V. Maheshwari K, Yang D, et al. Relationship between intraoperative hypotension, defined by either reduction from baseline or absolute thresholds, and acute kidney and myocardial injury after noncardiac surgery: A retrospective cohort analysis Anesth 2017;126:47-65.

7. Sessler DI. Khanna AK. Perioperative myocardial injury and the contribution of hypotension. Intensive Care Med 2018;44:811-22.

8. Monk TG. Bronsert MR, Henderson WG. Mangione MP, Sum-Ping ST. Bentt DR, et al. Association between intraoperative hypotension and hypertension and 30-day postoperative mortality in noncardiac surgery. Anesthesiology 2015;123:307-19.

9. Gan TJ. Soppitt A, Maroof M. el-Moalem H, Robertson KM. Moretti E, Dwane P. Glass PS. Goaldirected intraoperative fluid administration reduces length of hospital stay after major surgery Anesthesiology 2002;117:820-6. 
10. Wakeling HG. McFall MR, Jenkins CS. Woods WG, Miles WF. Barclay GR, Fleming SC. Intraoperative oesophageal Doppler guided fluid management shortens postoperative hospital stay after major bowel surgery. Br J Anaesth 2005;95:634-42.

11. Noblett SE. Snowden CP, Shenton BK. Horgan AF. Randomized clinical trial assessing the effect of Doppler-optimized fluid management on outcome after elective colorectal resection. Br J Surg 2006;93:1069-76.

12. Kamenik M. Kos D, Moller Petrun A. Haemodynamic stability during anaesthesia induction with propofol - impact of phenylephrine. A double-blind, randomised clinical trial. Signa Vitae 2018; $14(1): 20-6$.

13. Hahn RG. Arterial pressure and the elimination of crystalloid fluid: a population-based study. Anesth Analg. 2017; 124:1824-33.

14. Hahn RG. Understanding volume kinetics. Acta Anaesthesiol Scand. 2020;64(5):570-8.

15. Suedfeld S. Brechitz S, Wagner JY, et al. Post - induction hypotension and early intraoperative hypotension associated with general anaesthesia. British J Anaest 2017;119(1):57-64.

16. Struys MMRF. Coppens MJ, De Neve N, et al. Influence of administration rate on propofol plasmaeffect site equilibration. Anesthesiology 2007; 107: 386-96..

17. Chan VW. Chung FF. Propofol infusion for induction and maintenance of anaesthesia in elderly patients: recovery and haemodynamic profiles. J Clin Anesth 1996; 8: 317-23.

18. Pilge S. Zanner R, Schneider G. Blum J, Kreuzer M. Kochs EF. Time delay of index calculation: Analysis of cerebral state, bispectral, and narcotrend indices. Anesthesiology 2006; 104: 488-94.

19. White PF. Intravenous (non-opioid) anaesthesia. Seminars in Anesthesia. Perioperative Medicine Pain. 2005;24(2):101-7.

20. Möller Petrun A. Kamenik M. Bispectral index-guided induction of general anaesthesia in patients undergoing major abdominal surgery using propofol or etomidate: a double-blind, randomized, clinical trial. Br J Anaesth 2013;110(3):388-96.

21. Sessler DI. Bloomstone JA, Aronson S. Berry C, Gan TJ. Kellum JA, et al. Perioperative quality initiative consensus statement on intraoperative blood pressure, risk and outcomes for elective surgery. Br J Anaesth 2019;122:563-74.

22. Thiele RH. Nemergut EC, Lynch $\mathrm{C}$ 3rd. The clinical implications of isolated alpha-1 adrenergic stimulation. Anesthesia Analgesia. 2011;113(2):297-304.

23. Wodack KH, Graessler MF, Nishimoto SA, et al. Assessment of central hemodynamic effects of phenylephrine: an animal experiment. J Clin Monit Comput 2019;33:377-84.

24. Gelman S. Mushlin PS, Weiskopf RB. Catecholamine-induced Changes in the Splanchnic Circulation Affecting Systemic Hemodynamics. Anesthesiology 2004;100:434-9.

25. Gelman S. Bigatello L. The physiologic basis for goal - directed hemodynamic and fluid therapy: the pivotal role of the venous circulation. Can J Anesth 2018;65:295-308. 
26. Rebet $\mathrm{O}$. Andremont $\mathrm{O}$, Gérard JL, et al. Preload dependency determines the effects of phenylephrine on cardiac output in anaesthetised patients: A prospective observational study. Eur J Anaesthesiol 2016 Sep,33(9):638-44.

27. Jentzer JC. Coons JC, Link CB. Schmidhofer M. Pharmacotherapy update on the use of vasopressors and inotropes in the intensive care unit. J Cardiovasc Pharmacol Ther 2015;20(3):249-60.

28. Hasanin A, Habib S. Abdelwahab Y, et al. Variable versus fixed-rate infusion of phenylephrine during caesarean delivery: a randomized controlled trial. BMC Anesthesiol 2019;19(1):197.

29. Oh A. Hwang J, Song I, et al. Influence of the timing of administration of crystalloid on maternal hypotension during spinal anesthesia for caesarean delivery: preload versus coload. BMC Anesthesiol 2014;14:36.

30. Ni HF. Liu HY, Zhang J. Peng K, Ji FH. Crystalloid Coload Reduced the Incidence of Hypotension in Spinal Anesthesia for Caesarean Delivery, When Compared to Crystalloid Preload: A Meta-Analysis. Biomed Res Int. 2017;2017:3462529..

31. Wolff CB. Green DW. Clarification of the circulatory patho-physiology of anaesthesia - Implications for high-risk surgical patients. International journal of surgery 2014; 12(12):1348-56.

32. Jacobs R. Lochy S, Malbrain MLNG. Phenylephrine-induced recruitable preload from the venous side. J Clin Monit Comput 2019;33(3):373-6.

\section{Tables}

Table 1: Patient characteristics and comorbidities for the test and the control group. Values are mean (SD) or number of cases (percent).

\begin{tabular}{|lllll|}
\hline Variable & Control group N=19 & Test group N=20 & p \\
\hline Age $(\mathbf{y r})$ & $69.6(8.9)$ & $67.6(7.9)$ & 0.45 \\
\hline Gender $(\mathrm{m} / \mathrm{f})$ & & $10 / 9(52.6 \% / 47.4 \%)$ & $12 / 8(60.0 \% / 40.0 \%)$ & 0.74 \\
\hline Propofol dosage & & $86.0(33.4)$ & $75.3(24.7)$ & 0.26 \\
\hline Comorbidities & Hypertension & $17(89 \%)$ & $18(90 \%)$ & 1.0 \\
\cline { 2 - 5 } & Diabetes mellitus & $8(42 \%)$ & $4(20 \%)$ & 0.34 \\
\cline { 2 - 5 } & St. post TIA & $1(5 \%)$ & $0(0 \%)$ & 1.0 \\
\cline { 2 - 5 } & St. post AMI & $1(5 \%)$ & $2(10 \%)$ & 1.0 \\
\cline { 2 - 5 } & Hypothyroidism & $2(10 \%)$ & $2(10 \%)$ & 1.0 \\
\cline { 2 - 5 } & Depression & $1(5 \%)$ & $1(5 \%)$ & 1.0 \\
\hline
\end{tabular}


Table 2: Haemodynamic data for both study groups.

Values are mean ( $\pm \mathrm{SD})$, Units are: $\mathrm{Cl}((\mathrm{l} / \mathrm{min} / \mathrm{m} 2)$, SVRI (dynes.s/cm5/m2), MAP $(\mathrm{mmHg}), \mathrm{SVI}(\mathrm{ml} / \mathrm{m} 2)$, HR (1/min), SVV (\%) 


\begin{tabular}{|c|c|c|c|c|c|}
\hline & & Initial value & 6 minutes & 9 minutes & 18 minutes \\
\hline \multicolumn{6}{|l|}{$\mathrm{Cl}$} \\
\hline & Control group & $3,25( \pm 0,83)$ & $2,34( \pm 0,67)$ & $2,43( \pm 0,76)$ & $1,94( \pm 0,52)$ \\
\hline & Test group & $3,12( \pm 0,74)$ & $2,34( \pm 0,62)$ & $2,62( \pm 0,80)$ & $2,19( \pm 0,59)$ \\
\hline & $\mathrm{p}$ & & $0,450^{1-6}$ & $0,130^{1-9}$ & $0,052^{1-18}$ \\
\hline & $\mathrm{p}$ & & & $0,255^{6-9}$ & $0,256^{9-18}$ \\
\hline \multicolumn{6}{|l|}{ SVRI } \\
\hline & Control group & $2290( \pm 560)$ & $2684( \pm 609)$ & $2733( \pm 746)$ & $2960( \pm 759)$ \\
\hline & Test group & $2482( \pm 746)$ & $2611( \pm 586)$ & $2825( \pm 693)$ & $2919( \pm 754)$ \\
\hline & $\mathrm{p}$ & & $0,120^{1-6}$ & $0,782^{1-9}$ & $0,421^{1-18}$ \\
\hline & $\mathrm{p}$ & & & $0,170^{6-9}$ & $0,577^{9-18}$ \\
\hline \multicolumn{6}{|l|}{ MAP } \\
\hline & Control group & $94,9( \pm 14,7)$ & $81,7( \pm 14,9)$ & $85,6( \pm 20,9)$ & $75,0( \pm 11,7)$ \\
\hline & Test group & $98,6( \pm 12,1)$ & $79,8( \pm 11,1)$ & $94,3( \pm 14,5)$ & $82,5( \pm 12,7)$ \\
\hline & $\mathrm{p}$ & & $0,286^{1-6}$ & $0,240^{1-9}$ & $0,096^{1-18}$ \\
\hline & $\mathrm{p}$ & & & $0,020^{6-9}$ & $0,169^{9-18}$ \\
\hline \multicolumn{6}{|l|}{ SVI } \\
\hline & Control group & $48,4( \pm 11,0)$ & $38,9( \pm 12,4)$ & $37,0( \pm 11,4)$ & $35,4( \pm 12,2)$ \\
\hline & Test group & $44,7( \pm 9,3)$ & $38,3( \pm 9,4)$ & $41,6( \pm 10,9)$ & $40,8( \pm 9,5)$ \\
\hline & $\mathrm{p}$ & & $0,102^{1-6}$ & $0,002^{1-9}$ & $0,006^{1-18}$ \\
\hline & $\mathrm{p}$ & & & $0,006^{6-9}$ & $0,428^{9-18}$ \\
\hline \multicolumn{6}{|l|}{ HR } \\
\hline & Control group & $67,9( \pm 12,4)$ & $62,2( \pm 12,4)$ & $67,3( \pm 13,2)$ & $56,9( \pm 11,7)$ \\
\hline & Test group & $70,3( \pm 10,9)$ & $61,6( \pm 9,1)$ & $63,2( \pm 9,4)$ & $53,7( \pm 7,2)$ \\
\hline & $\mathrm{p}$ & & $0,337^{1-6}$ & $0,046^{1-9}$ & $0,041^{1-18}$ \\
\hline & $\mathrm{p}$ & & & $0,236^{6-9}$ & $0,621^{9-18}$ \\
\hline \multicolumn{6}{|l|}{ sVV } \\
\hline & Control group & $0,105( \pm 0,060)$ & $0,096( \pm 0,051)$ & $0,141( \pm 0,060)$ & $0,122( \pm 0,074)$ \\
\hline
\end{tabular}




\begin{tabular}{|lllll|}
\hline Test group & $0,091( \pm 0,047)$ & $0,086( \pm 0,047)$ & $0,083( \pm 0,062)$ & $0,078( \pm 0,043)$ \\
\hline$p$ & $0,467^{1-6}$ & $\mathbf{0 , 0 0 7 7 ^ { 1 - 9 }}$ & $\mathbf{0 , 0 3 6 ^ { 1 - 1 8 }}$ \\
\hline$p$ & & $\mathbf{0 , 0 0 7 ^ { 6 - 9 }}$ & $\mathbf{0 , 4 3 0}{ }^{\text {9-18 }}$ \\
\hline
\end{tabular}

\section{Figures}

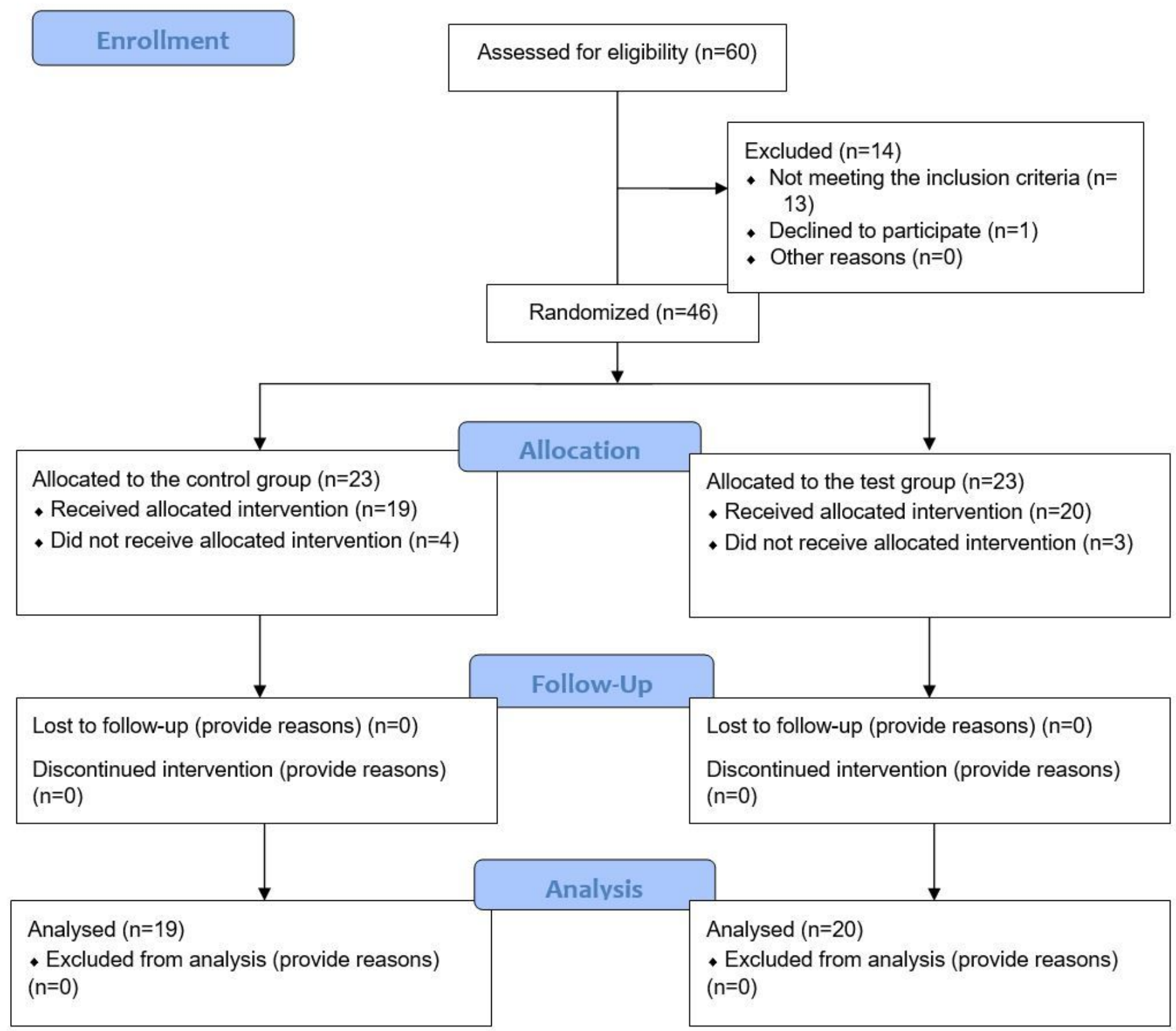

\section{Figure 1}

Flowchart of the conduct of the study. 

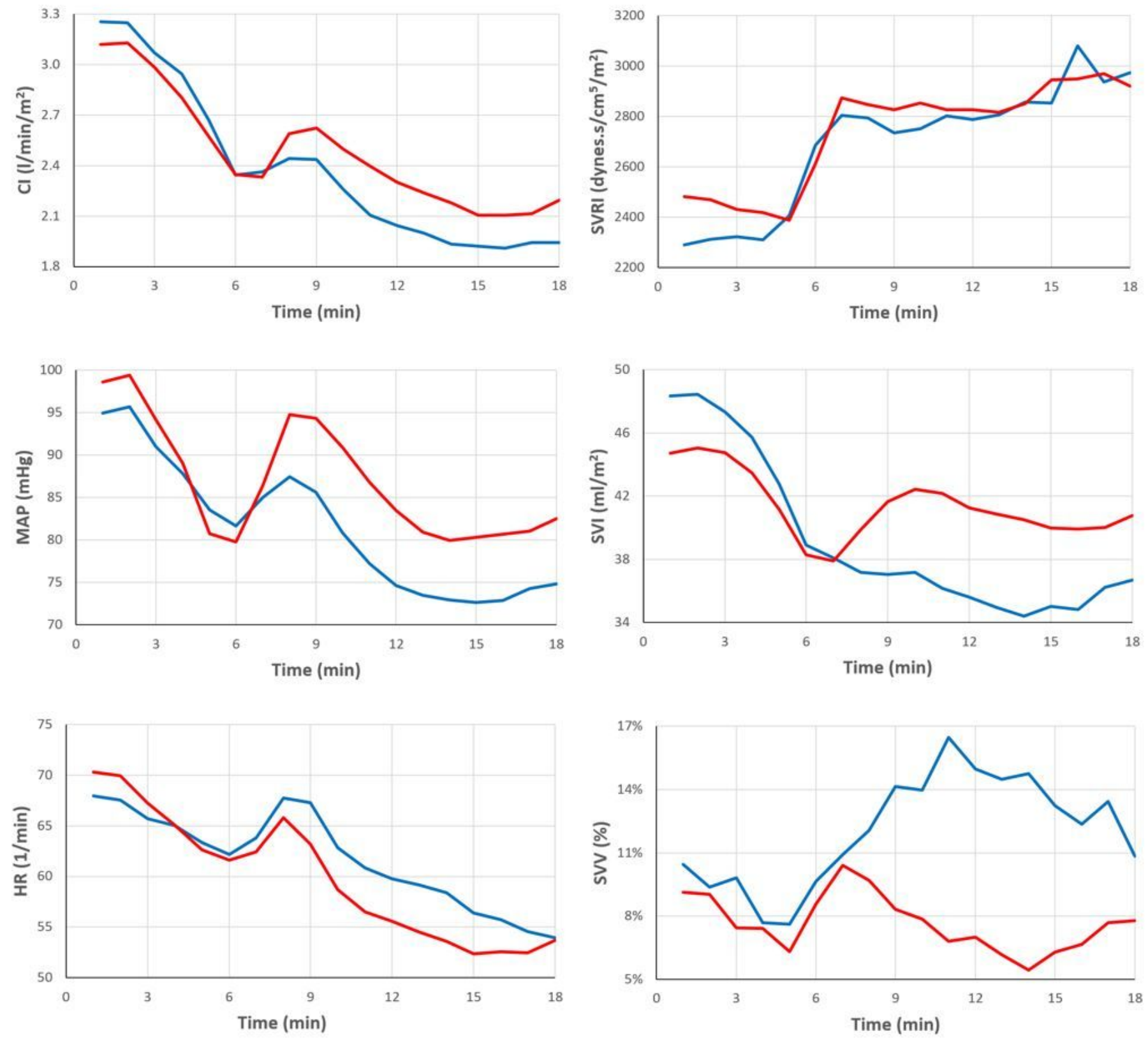

Figure 2

Haemodynamic data development for both study groups. Blue - Control group, Red - Test group. 